\title{
Árion: A Blockchain Framework for Traceability in Supply Chain Management
}

\author{
Edivaldo M. F. J. Júnior ${ }^{1}$, Manoel C. M. Neto ${ }^{1}$, Allan E. S. Freitas ${ }^{1}$ \\ ${ }^{1}$ Programa de Pós-Graduação em Engenharia de Sistemas e Produtos \\ Instituto Federal da Bahia (IFBA) \\ Campus de Salvador, 40301-015, Salvador - BA - Brazil \\ \{edivaldojunior, manoelnetom, allan\}@ifba.edu.br
}

\begin{abstract}
A complex web of relationships provides goods for manufacturing, assembling, and delivering final products known as the supply chain. Emerging technologies have been used in supply chain systems in order to provide traceability. However, these systems tend to be centralized, monopolistic, and asymmetric. As a consequence, they may result in trusting problems, such as fraud, corruption, and tampering. Blockchain technology provides a new approach for information systems based on decentralization, that can apply for supply chain systems. This work presents a Blockchain-based framework for developing applications that provide such traceability for supply chain management.
\end{abstract}

\section{Introduction}

Hundreds of years ago, supply chains were fairly simple. Mines and farms provided natural resources to skilled craftsman like blacksmiths and tailors who then created and sold goods. Nowadays, supply chains are much more complicated, fragmented and difficult to understand.

Today's supply chains are so complex that even big industry players have difficulty tracking how their goods get made [Swan 2015]. In order to solve some problems that come with this complexity, such as supply chain visibility and traceability, many systems have been developed. However, these systems typically store information in standard databases controlled by service providers. This centralized data storage becomes a single point of failure and risks tampering [Tian 2017].

Blockchain could make supply chain management (SCM) simpler and more transparent. The idea is to create a single source of information about products and supply chain via global ledger. Each component would have its own entry on the blockchain that gets tracked over time. The end result is once the clients receive their products they could track every piece back to its manufacturer [Greve et al. 2018].

This would reduce the need for large contract invoices on the back-and-forth of refund requests for faulty components. Those same smart contracts could assist with shipping and logistics tracking valuable products as they travel around the world. Using blockchain, companies can finally have a complete picture of their products at every stage in the supply chain, bringing transparency to the production process while reducing the cost of manufactured goods [Swan 2015].

This work presents Árion, a generic framework intended to be used in any kind of supply chain correlated to assets and products. 


\section{Blockchain}

Blockchain can be considered as a public ledger, in which all committed transactions are stored in a block chain [Zheng et al. 2016]. For [Swan 2015], Blockchain is in a position to become the fifth disruptive computing paradigm after mainframes, PCs, Internet and mobile/ social networks. Blockchain technology has critical features, such as decentralization, persistence, anonymity and auditability [Zheng et al. 2016].

\subsection{Public Blockchain Versus Private Blockchain}

On a public blockchain, any person can participate without a specific identity. They can be audited by anyone, and each node has as much transmission power as any other. As long as each node meets protocol-specific stipulations, their transactions can be validated and thus added to the chain [Greve et al. 2018]. Private blockchains, on the other hand, perform a blockchain between a set of known and identified participants. A private blockchain provides a way to protect the interactions between a group of entities that have a common goal but that don't totally trust each other [Swan 2015].

\subsection{Smart Contracts}

A smart contract is a computerized transaction protocol that executes the terms of a contract [Szabo 1997]. The term smart contract (SC) means: "an internal transaction protocol format that executes the terms of a contract. Their overall goals are ensure common contractual conditions, minimize malicious and accidental exceptions and the need for reliable intermediaries. Related economic objectives include reducing fraud losses, arbitration and execution costs, and other transaction costs." [Szabo 1997].

\section{Supply Chain Management}

There are billions of products being manufactured every day through complex supply chains that can extend to all parts of the world. However, tracing good flows from harvesting and manufacturing to the final consumer is hard [Galvez et al. 2018]. Traceability is one of the key challenges encountered in the business world. These systems typically store information in standard databases controlled by service providers. This centralized data storage becomes a single point of failure and tampering risks. As a consequence, these systems result in trusting problems, such as fraud, corruption, and tampering. Likewise, as a single point of failure, a centralized system is vulnerable to collapse [Tian 2017].

Nowadays, Blockchain presents a whole new approach based on decentralization, enabling end-to-end traceability, allowing consumers to access the asset's history of these products through a software application [Galvez et al. 2018]. Supply chain management requires to control who can write and read data to/from the Blockchain. In order to do that, the first step is identity. In the SCM context, the peers are known and the system needs to know who a user is, to define rules about what data they can commit, and what data they can consume from the ledger. So, in a corporate case scenario, Blockchain for the business, a private Blockchain provides this needed characteristic.

\section{Related Work}

In order to solve some problems with Supply Chain traceability centralized, monopolistic, and asymmetric systems, many internet of things (IoT) technologies has been applied. 
However, these technologies do not guarantee that the information shared by supply chain members in the traceability systems can be trusted [Tian 2017].

In [Tian 2017], it is proposed a system that combines HACCP (Hazard Analysis and Critical Control Point, a food safety protocol), Blockchain and IoT in order to provide food safety traceability. Each product has a unique digital cryptographic identifier that connects the physical items to their virtual identity in the system. This virtual identity can be seen as the product information profile.

The Everledger Diamonds project provides a Blockchain based solution to facilitate tracking from mine to consumer, enabling easier compliance against increasingly strict measures from diamonds produced [Crosby et al. 2016].

These projects are focused on specific products only and are closed projects. Still, there is a general lack of standards for implementation of a Blockchain approach for traceability. A Blockchain must be universal and adaptable to specific situations [Valenta and Sandner 2017]. In addition, the need to agree on a particular type of Blockchain to be used puts the parties under pressure.

Our work is intended to provide a Blockchain based framework in order to facilitate the development of applications for traceability in supply chain management.

\section{Proposed Framework}

The main objective of this work is to create a generic framework for Supply Chain Management (SCM). Árion project is divided into three main modules described below: Frond-End WebApp, Back-End WebApp, and Data Storage. Figure 1 shows the application architecture and its components. An SCM platform relies on three main items: assets, steps, and actors. Our approach is based on this triad, that must be defined on the creation of a new supply chain.

\subsection{Frond-End WebApp}

Front-End WebApp is a client-server application which the client runs in a web browser. The Front-End Webapp is divided into two main blocks and these are classified according to the interactions: User Interaction Modules and Backend Interaction Services. User Interaction modules are responsible for providing web pages that will be rendered on client's web browser. The backend interactions happen via a set of services.

\subsection{Back-End WebApp}

BackEnd - WebApp is a Middleware that runs on the server facilitating the client-server connectivity, forming a middle layer between the app and the network. It contains the logic to send the appropriate data back to the applicant. This module is composed by the API Gateway, Service Layer and Resource Locator. API Gateway is a managed service that enables easily create, publish, maintain, monitor and secure REST APIs. The service Layer defines its set of available operations from the perspective of interfacing client layers. The service layer acts as an orchestrator, controlling the flow of incoming and outcoming information requests and responses. Resource locators are components that abstracts the persistence layer. Their job is to provide an object that can help services to discover and persist information from/to the Data Storage Module. 


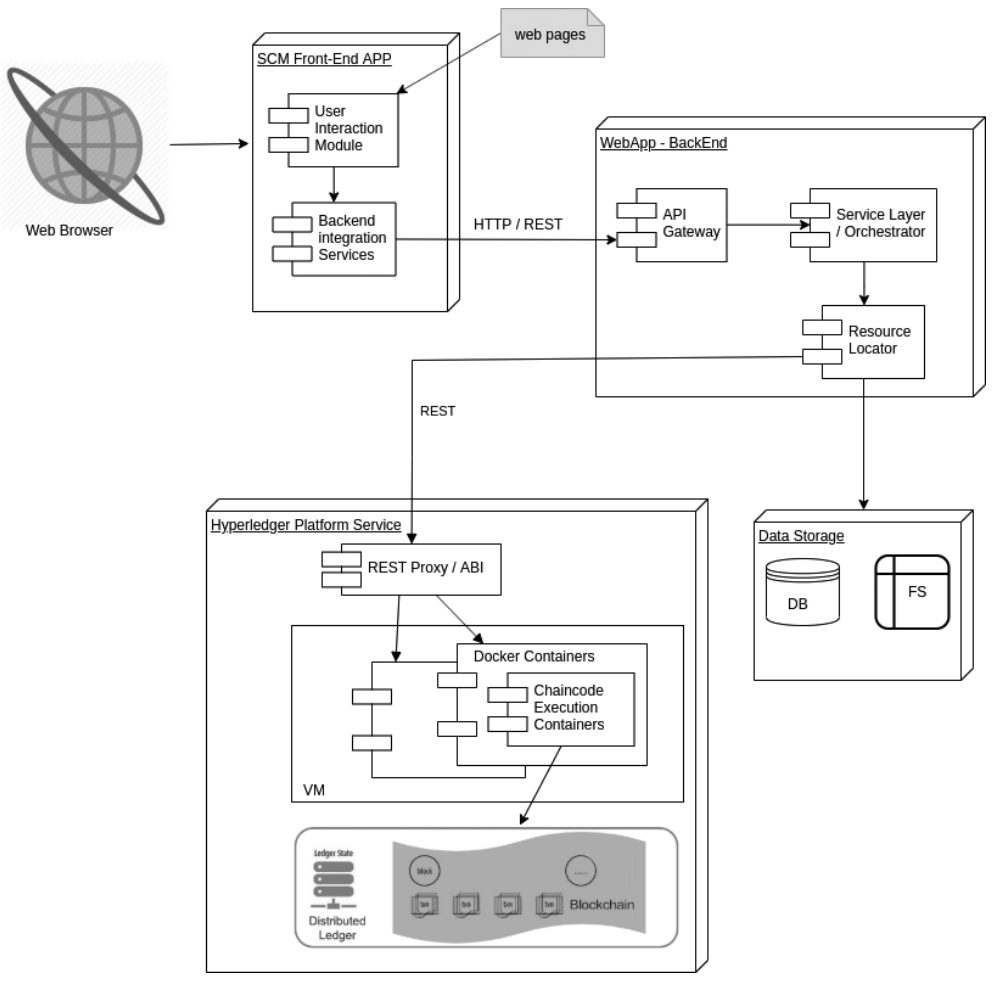

Figure 1. Árion Application architecture

\subsection{Data Storage}

Árion uses three applications as data storage: Blockchain, Cloud file system, and relational database. Blockchains grow continuously because of the amount of data and code in them, which is unchanging. Therefore, an important design decision is to choose which data and calculations to keep in and out of the chain. A cloud file system is a tiered storage system that provides shared access to file data. A relational database is a set of formally described tables from which data can be accessed or reassembled in many different ways without having to reorganize the database tables.

\subsubsection{Blockchain and Chaincode}

The platform uses Blockchain to supply chain management tracking parts and service provenance, ensuring authenticity of goods, block counterfeits and reducing conflicts. This usually involves a limited and known number of actors, suggesting use of a permissioned Blockchain, that is, a Blockchain where all nodes must be allowed to be part of the system. To implement that, Hyperledger Fabric is used [Cachin et al. 2016].

Hyperledger Fabric implements smart contracts through chaincode. In general, a smart contract defines the transaction logic that controls the lifecycle of a business object contained in the world state. It is then packaged into a chaincode which is then deployed to a Blockchain network. So, smart contracts rule transactions, whereas chaincode rules how smart contracts are packaged for deployment.

Before businesses transact with each other, they must define a common set of 
contracts covering common terms, data, rules, concept definitions, and processes. Taken together, these contracts lay out the business model that govern all of the interactions between transacting parties. A smart contract defines the rules between different organizations in executable code. Applications invoke a smart contract to generate transactions that are recorded on the ledger.

\section{Implementation Details}

Our chaincode is written in Golang and provides all contracts needed to proceed traceability in our application. The first step is to create a JSON config file providing all information about these three items: the assetId, a list of actors and a list of ordered steps. Our chaincode processes this file through initLedger and createNewAsset functions. Front-end WebApp enables a user to define settings through a Configuration Page, adding these to the configuration file.

Assets, asset items, steps and actors are described as structs. Query methods are responsible for interact with the information of any item in the Blockchain. The function main invokes the initLedger, reads the configuration files and raises the platform enabling users to interact with the Blockchain via exposing its API. When creating an asset item, an AssetItemId is generated. Each entity in the chain will have its unique entity ID and timestamp when it starts processing the transaction. By querying AssetItemId, the user can easily track the current transaction information and status. Finally, completed all steps, the Blockchain will update deliverDate and mark the status as completed once the final actor has received the order. ChangeAssetItemOwner is the method called to update an asset item when it is moved from a step to another. It updates the CurrentOwnerId, the ProcessDate, information about prices and many other details of the transactions by the key/value map aditionalInfo.

Figure 2 shows the interaction flow from users with Árion platform. Initially an admin persona creates and configure the SCM adding information about the steps and the users. After that, the admin can activate this SCM and from that point the actors can interact with the SCM to provide information about an asset item and also move this asset item through the supply chain. From that point too, any user can track an asset item to get information about the required good.

\section{Conclusion}

Although, some companies have launched pilot projects using Blockchain technology to manage their supply chains, no detailed information on the technical implementation of such projects has been reported. Either way, the retail industry has the potential to use this technology to improve traceability. Even if some properties of Blockchain implementation may be useful for supply chain management, there are still a few uses to support this claim.

In this paper, we proposed a framework for new decentralized traceability systems based on Blockchain technology. This system will deliver online information to all supply chain members on the safety status of goods, providing a more secure, distributed, transparent, and collaborative approach to supply chain management. The framework can significantly improve the development time of Supply Chain Management applications, and provide efficiency and transparency for product management on a supply chain. In a 


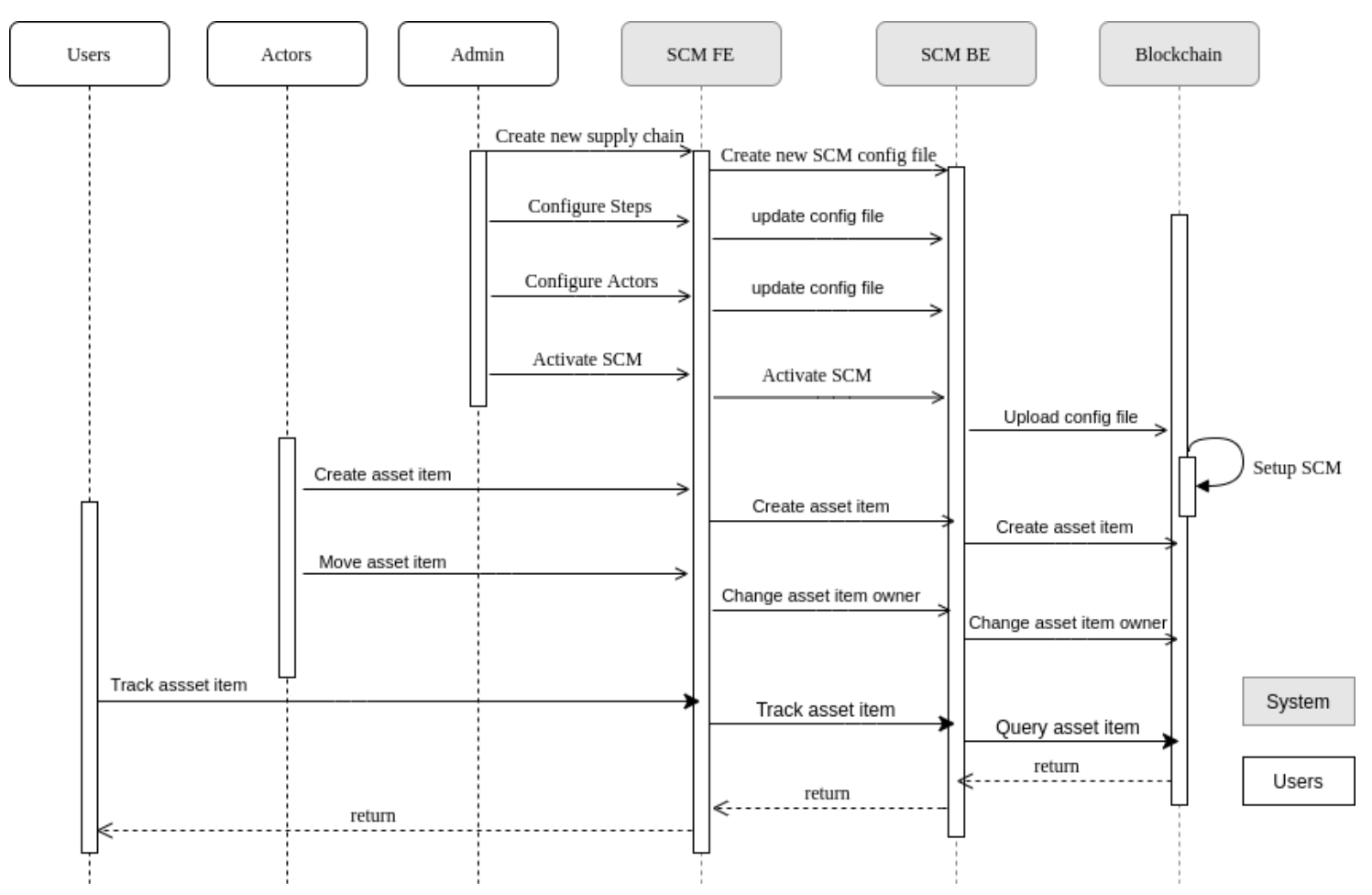

Figure 2. SCM User flow

future work, we will present a proof of concept that exercises the use of proposed framework.

\section{References}

Cachin, C. et al. (2016). Architecture of the hyperledger blockchain fabric. In Workshop on distributed cryptocurrencies and consensus ledgers, volume 310, page 4.

Crosby, M., Pattanayak, P., Verma, S., Kalyanaraman, V., et al. (2016). Blockchain technology: Beyond bitcoin. Applied Innovation, 2(6-10):71.

Galvez, J. F., Mejuto, J., and Simal-Gandara, J. (2018). Future challenges on the use of blockchain for food traceability analysis. TrAC Trends in Analytical Chemistry.

Greve, F., Sampaio, L., ABIJAUDE, J., COUTINHO, A., VALCY, I., and Queiroz, S. (2018). Blockchain e a revolução do consenso sob demanda. Livro de Minicursos do SBRC, 1:1-52.

Swan, M. (2015). Blockchain: Blueprint for a new economy. " O’Reilly Media, Inc.”.

Szabo, N. (1997). The idea of smart contracts. Nick Szabo's Papers and Concise Tutorials.

Tian, F. (2017). A supply chain traceability system for food safety based on haccp, blockchain \& internet of things. In 2017 International Conference on Service Systems and Service Management, pages 1-6. IEEE.

Valenta, M. and Sandner, P. (2017). Comparison of ethereum, hyperledger fabric and corda. [ebook] Frankfurt School, Blockchain Center.

Zheng, Z., Xie, S., Dai, H.-N., and Wang, H. (2016). Blockchain challenges and opportunities: A survey. Work Pap.-2016. 